ERRATUM

doi:10.1038/nature13571

\title{
Erratum: CTP synthase 1 deficiency in humans reveals its central role in lymphocyte proliferation
}

Emmanuel Martin, Noé Palmic, Sylvia Sanquer,

Christelle Lenoir, Fabian Hauck, Cédric Mongellaz, Sylvie Fabrega, Patrick Nitschké, Mauro Degli Esposti, Jeremy Schwartzentruber, Naomi Taylor, Jacek Majewski, Nada Jabado, Robert F. Wynn, Capucine Picard, Alain Fischer, Peter D. Arkwright \& Sylvain Latour

Nature 510, 288-292 (2014); doi:10.1038/nature13386

Owing to a production error, the vertical axis of the right panel of Fig. $3 g$ was misaligned. The correct panel is shown below as Fig. 1 of this Erratum. In addition, the legends for Fig. 2 and Extended Data Fig. 3 should read "Induction of CTPS1 expression during T- and B-cell activation and defective proliferation of activated CTPS1-deficient T and B cells" and "Induction of CTPS1 expression in activated B cells and inhibitors of CTPS1 expression in activated T cells", respectively.

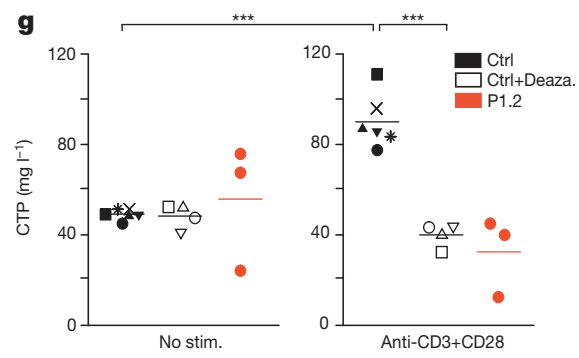

Figure $1 \mid$ This is the corrected Fig. 3g of the original Letter. 